\title{
Editorial
}

\section{Statistical Models in Finance and Insurance}

\author{
Arkady Shemyakin ${ }^{\mathrm{a}}$ and Vladimir Ladyzhets ${ }^{\mathrm{b}}$

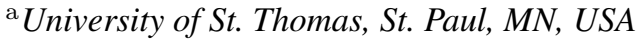 \\ ${ }^{\mathrm{b}}$ University of Connecticut, Stanford, CT, USA
}

The special issue of Model Assisted Statistics and Applications, 12 (4) 2017, is dedicated to Statistical Models in Finance and Insurance. As Guest Editors, we would like to introduce seven papers comprising the issue and present the unifying ideas explaining the choice of the content.

Our goal for the special issue is offering to the readers a variety of topics representing a wide range of applications of statistical methods to insurance and finance, requiring new methodological approaches. Classical development of financial and actuarial mathematics in the 20-th century including such fundamental achievements as Markowitz model, CAPM, Black-Scholes formula, Buhlmann's credibility theory, CDO pricing using Gaussian copulas and others, was effectively based on mean and covariance, thus, heavily relying on accuracy of an open or hidden assumption of normality of underlying variables. Statistical analysis of financial variables emphasized the estimation of distribution moments, and the study of dependence between variables was mostly reduced to correlation analysis. This approach worked successfully for a while. However, one of the principal challenges of financial and insurance risk modeling is an extreme fluidity of their universe, at least in comparison with the surrounding physical world. When market agents trust the modeling assumptions, models work. When this trust is shaken, models cease to reflect the reality. Realities of the last decades and the turn of the 20-th century in a most painful way demonstrated the inadequacy of the classical approach and a need for new developments. The new financial environment called for a new mathematical and statistical methodology. Introducing asymmetric and tail-heavy distribution models for risk variables and going beyond correlation in description of their association becomes a staple of modern quantitative analysis. Therefore, the emphasis in this issue is made on the modern understanding of financial and insurance practice beyond the standard set of assumptions requiring normality of key modeling variables. It calls for the development of new methods under somewhat more complicated assumptions reflecting a more realistic view of the financial and insurance world.

We introduce seven papers, with the order of a particular article suggested by similarity of applications rather than by a particular statistical technique.

The two opening articles deal with the models of market risks reflecting the realities of post-Black-Scholes statistical methods. The first paper by Chung and Niu "Financial Volatility Estimation Using Functional Gradient Descent Algorithm" introduces a new semiparametric model of volatility in financial time series, presenting an alternative to existing stochastic volatility models. De Lara-Tuprio and Sumalpong in "British Put Option on Stock under Stochastic Interest Rate” derive a closed-form pricing formula under stochastic interest rate (Vasicek model), while previous results are confined to constant interest rate. Assuming the stochastic nature of volatility and interest rates provides a more realistic modeling structure for practical applications, expanding classical methodology.

Two other papers directly address credit risk issues arising in financial and insurance applications. The study of Penikas and Ermolova "PD-LGD Correlation Study: Evidence from Russia Corporate Bond Market" demonstrates that the correlation between the probability of default (PD) and loss-given-default (LGD) depends on the market being studied, with different effects observed for developed and developing countries, thus, expanding traditional credit risk models for PD and LGD. Galloway et al. in "Time-to-default Analysis of Mortgage Portfolios" use Bayesian mixture models to analyze defaults in residential mortgages in the U.S. This study brings about some interesting observations regarding the latent segmentation of prime and subprime portfolios. 
Kreinin et al. in “Correlated Multivariate Poisson Processes and Extreme Measures” develop a multi-dimensional generalization of backward simulation approach recently proposed by one of the authors for two dimensions. This approach allows for modeling mixed Poisson process and analyzing the connection to the extreme measures describing the joint distribution of the processes at the end of the time horizon. The methodology presented in the paper brings a solution to one of the long standing challenges in running credit risk and operational risk stress test scenarios: simulation of multivariate Poisson processes with an arbitrary correlation matrix (including one with negative values).

Goldberg in "Anti-robust and Tonsured Statistics" suggests a new (though natural) statistical tool providing a descriptive measure for non-linear dependence, especially designed to capture tail association between financial variables. This approach introduces "tonsuring", which in many contexts can provide an attractive alternative to more traditional correlation measures. This tool is suggested by a practitioner to practitioners. The paper deals with interesting novel concepts of financial statistics rather than specific applications, and is more related to the field of general methodology, though it can be naturally applied to portfolio risks. However, further justification of the validity of this approach and study of the mathematical properties of "tonsuring" statistic seems to be an interesting direction of development in statistical theory.

Kravchenko et al. in "Determinants of Growth of Small High-tech Companies in Transition Economies" use a survey of small high-tech service and manufacturing businesses in Russia to establish environmental, supply and demand factors of company growth which could explain and predict their further development. The authors utilize generalized linear modeling procedures providing an extension of ordinary least squares regression to an essentially non-Gaussian environment. An attractive feature of the paper is the effort to construct an investment rating based on measurable variables suggested as proxies of financial success.

The initial portfolio contained over twenty submissions, and it was no easy task to select the best papers. We would like to thank all our colleagues, both from academia and business, for serving as referees for the issue. They have made this selection possible and contributed immensely to the refinement of the materials accepted for publication. 